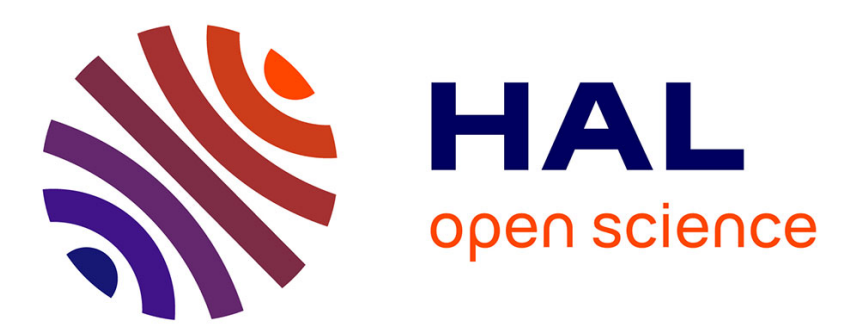

\title{
Use of FDA approved therapeutics with hNTCP metabolic inhibitory properties to impair the HDV lifecycle
}

\author{
Matthieu Blanchet, Camille Sureau, Patrick Labonté
}

\section{To cite this version:}

Matthieu Blanchet, Camille Sureau, Patrick Labonté. Use of FDA approved therapeutics with hNTCP metabolic inhibitory properties to impair the HDV lifecycle. Antiviral Research, 2014, 106, pp.111-115. 10.1016/j.antiviral.2014.03.017 . hal-01199003

\section{HAL Id: hal-01199003 \\ https://hal.science/hal-01199003}

Submitted on 14 Sep 2015

HAL is a multi-disciplinary open access archive for the deposit and dissemination of scientific research documents, whether they are published or not. The documents may come from teaching and research institutions in France or abroad, or from public or private research centers.
L'archive ouverte pluridisciplinaire HAL, est destinée au dépôt et à la diffusion de documents scientifiques de niveau recherche, publiés ou non, émanant des établissements d'enseignement et de recherche français ou étrangers, des laboratoires publics ou privés. 


\title{
Use of FDA approved therapeutics with hNTCP metabolic inhibitory properties to impair the HDV lifecycle
}

Matthieu Blanchet ${ }^{\mathrm{a}}$,

Camille Sureau

Patrick Labonté,

doi:10.1016/.antiviral.2014.03.017

Highlights

Development and characterization of a Huh7 cell line with high expression of hNTCP.

Identification of three FDA approved therapeutics with antiviral effect against HDV.

We support the relationship between hNTCP metabolic and viral receptor function.

\begin{abstract}
Worldwide there are approximately 240 million individuals chronically infected with the hepatitis B virus (HBV), including 15-20 million coinfected with the hepatitis delta virus (HDV). Treatments available today are not fully efficient and often associated to important side effects and development of drug resistance. Targeting the HBV/HDV entry step using preS1-specific lipopeptides appears as a promising strategy to block viral entry for both HBV and HDV (Gripon et al., 2005; Petersen et al., 2008). Recently, the human Sodium Taurocholate Cotransporting Polypeptide (hNTCP) has been identified as a functional, preS1-specific receptor for HBV and HDV. This groundbreaking discovery has opened a very promising avenue for the treatment of chronic HBV and HDV infections. Here we investigated the ability of FDA approved therapeutics with documented inhibitory effect on hNTCP cellular function to impair viral entry using a HDV in vitro infection model based on a hNTCP-expressing Huh7 cell line. We demonstrate the potential of three FDA approved molecules, irbesartan, ezetimibe, and ritonavir, to alter HDV infection in vitro.
\end{abstract}




\section{Abbreviations}

- HDV, hepatitis delta virus;

- HBV, hepatitis B virus;

- hNTCP, human Sodium Taurocholate Cotransporting Polypeptide;

- NUC, nucleoside analogues;

- CsA,cyclosporine A;

- BA, bile acid

Keywords

- Hepatitis delta virus;

- Hepatitis B virus;

- hNTCP;

- Infection;

- FDA approved therapeutics;

- Antivirals

Despite of the availability of an efficient vaccine, approximately 240 million people are chronically infected with hepatitis B virus (HBV) worldwide (Ott et al., 2012). HBV chronic carriers may progress to severe liver disease, including cirrhosis and hepatocellular carcinoma (Ganem and Prince, 2004). Among HBV carriers, 15-20 millions are coinfected with the hepatitis delta virus (HDV) (Pascarella and Negro, 2011), a defective virus that uses the envelope of HBV to complete its lifecycle. Chronic infection with both HBV and HDV often results in more severe pathogenesis (Pascarella and Negro, $\underline{2011}$ and Smedile et al., 1981). For both viruses, current antiviral treatments lack efficiency and are generally unable to trigger viral clearance. The failure to eliminate the HBV infection is assumed to be due to the high stability the covalently closed circular HBV DNA (cccDNA) in the nucleus of infected hepatocytes. Pegylated-interferon presents both interesting direct antiviral activity and immune-stimulatory properties (Zoulim, 2011) but is accompanied by side effects and an efficiency limited to a minority of patients (Lok and McMahon, 2009). Nucleoside analogues (NUC) are also of interest for the treatment of HBV chronic infections, but their use can elicit adaptive mutations conferring drug resistance (Zoulim, 2011). In regard to HDV chronic infections, available treatments, mainly based on Pegylated interferon, are not satisfactory (Pascarella and Negro, 2011). A new approach targeting the viral entry step using pre-S1 lipopeptides that 
compete with infectious virions for receptor binding appears as a promising strategy (Gripon et al., 2005 and Petersen et al., 2008).

Recently the human Sodium Taurocholate Cotransporting Polypeptide (hNTCP) has been identified as a functional pre-S1-specific receptor of HBV and HDV (Yan et al., 2012), opening a promising avenue in the development of antivirals. FDA approved drugs that inhibit the metabolic function of the hNTCP are available (Dong et al., 2013), including cyclosporine A (CsA), which block bile acid (BA) transport function of hNTCP (Nkongolo et al., 2013 and Watashi et al., 2013). One can see an advantage in using antiviral drugs that target cellular proteins in reducing the frequency of drug induced adaptive mutations leading to resistance (Provencher et al., 2004). We reasoned that the chemicals that inhibit hNTCP metabolic functions might also block its HBV/HDV receptor and thus selected several inhibitors of the hNTCP metabolic function for in vitro analysis of their antiviral potential. This hypothesis was comforted by several recent studies (Nkongolo et al., 2013, Watashi et al., 2013 and Yan et al., 2014). Irbesartan, an angiotensin II receptor antagonist used mainly for the treatment of hypertension, ezetimibe, a drug that lowers plasma cholesterol levels, and ritonavir, an antiretroviral drug from the protease inhibitor class used to treat HIV infection, were selected based on their potent inhibitory effect on hNTCP metabolic function (Dong et al., 2013). Omeprazole, a drug known as inefficient against hNTCP function, was selected as a negative control.

We first developed a Huh7 cell line that constitutively expresses the hNTCP (see material and methods in Supplementary materials). To ascertain hNTCP expression in Huh7 cells, we performed a relative quantification of the hNTCP mRNA in Huh7 and Huh7/hNTCP cell lines. A 1000-fold increase in expression was observed for the stable Huh7/hNTCP cell line compared to Huh7 cells (Fig. 1A). To control the relevance of our cell line toward viral infection, we inoculated Huh7 and Huh7/hNTCP cells with HDV virions bearing either the three HBV envelope proteins L-, M-, S-HBsAg ( $\left.\mathrm{HDV}_{\mathrm{LMS}}\right)$ or non infectious virions bearing only the small HBV envelope protein S-HBsAg $\left(\mathrm{HDV}_{\mathrm{s}}\right)$ as negative control. Virus-containing supernatants were produced as described previously (Blanchet and Sureau, 2006). Relative quantification by RT-qPCR (Taqman) showed no significant difference in the HDV RNA content between $\mathrm{HDV}_{\text {LMS }}$ and $\mathrm{HDV}_{S}$ preparations 
(Fig. 1B). Huh7 and Huh7/hNTCP cells were exposed to HDV $\mathrm{Lms}_{\text {L }}$ and $\mathrm{HDV}_{\mathrm{s}}$ for $20 \mathrm{~h}$, and further cultured for 6 days, before intracellular HDV RNA content was quantified. The signal obtained after inoculation of Huh7/hNTCP with HDV $_{\text {LMS }}$ was $\sim 1000$ times higher than the one obtained with Huh7 cells (Fig. 1C). Additionally, the viral RNA concentration in Huh7/hNTCP was $\sim 1000$ fold higher after inoculation with $\mathrm{HDV}_{\text {LMS }}$ as compared with $\mathrm{HDV}_{\mathrm{s}}$. (Fig. 1C). The cytotoxicity of chemicals was assessed as follows: cells were treated with increasing concentrations of drugs (up to $50 \mu \mathrm{M}$ ) for $20 \mathrm{~h}$, washed, and further incubated in fresh media for 6 days. An MTS assay revealed no cytotoxicity at the tested concentrations (Fig.

$\overline{1}^{\mathrm{A})}$.

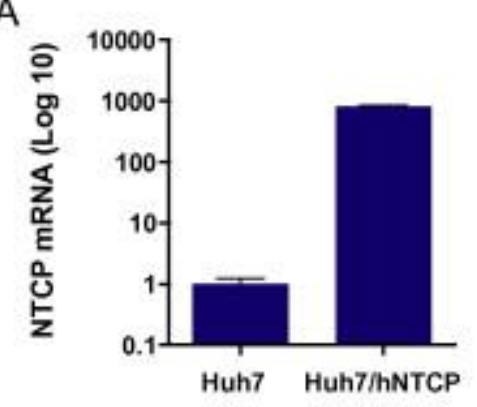

C

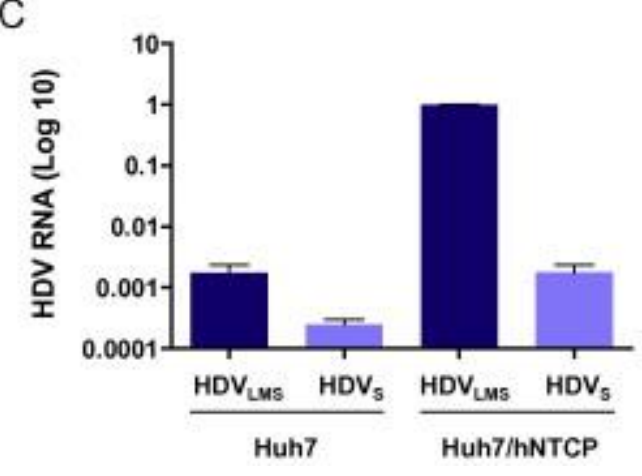

B

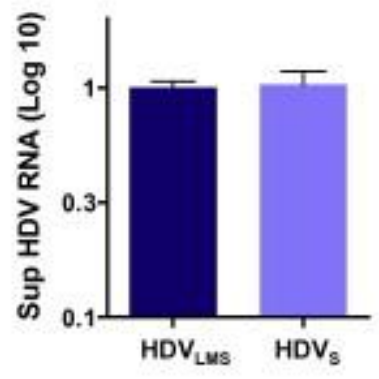

D

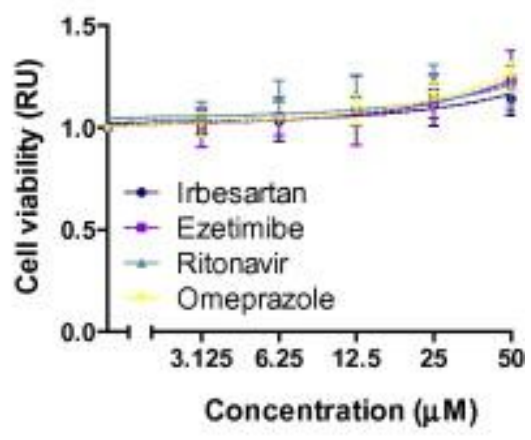

Fig. 1.

Susceptibility of hNTCP-expressing Huh7 cells to HDV infection. (A) Relative quantification of the hNTCP mRNA in Huh7/hNTCP and Huh7 cells was achieved by specific RT-qPCR after total RNA extraction and DNAse I treatment. (B) HDV LMs- $^{-}$and $\mathrm{HDV}_{\mathrm{s}}$-containing supernatants were produced by transfection of Huh7 cells with pSVLD3, a plasmid containing 3 copies of the HDV genome, and pT7HB2.7, a plasmid coding for the three HBV envelope proteins, or p123, a plasmid encoding the S-HBsAg (Blanchet and Sureau, 2006). Media were collected for 15 days and pooled. The relative HDV RNA concentrations in the 2 viral preparations were assessed by RT-qPCR (Taqman). (C) Huh7 and Huh7/hNTCP cells were incubated with $\mathrm{HDV}_{\text {LMS }}$ and $\mathrm{HDV}_{\mathrm{S}}$ preparations at a 
concentration of $\sim 125 \mathrm{GEq} / \mathrm{cell}$ in the presence of $4 \%$ PEG 8000 for $20 \mathrm{~h}$, extensively washed and further cultured for 6 days. Intracellular HDV RNA was quantified by RTqPCR (Taqman). (D) Cells were incubated with concentration up to $50 \mu \mathrm{M}$ of each compound for $20 \mathrm{~h}$, followed by extensive washing and further culture for 6 days in fresh media. Cell viability was assessed with a MTS assay. Viability is represented relatively to the non treated sample.

Figure options

The antiviral properties of the chemicals were monitored in different conditions: compounds were added for $20 \mathrm{~h}$ before, during, or after viral inoculation, as described inFig. 2A. Cells were washed and further cultured for 6 days. A significant reduction of intracellular HDV RNA concentration was observed upon simultaneous viral inoculation and treatment with irbesartan, ezetimibe, and ritonavir, $\left(\mathrm{EC}_{50}\right.$ of $\sim 9.4, \sim 10.4$, and $\sim 9.1 \mu \mathrm{M}$, respectively) as shown in Fig. 2. These effects were shown to be additive (Fig. 4A). Drug treatment prior to or after viral inoculation did not demonstrate any significant reduction of intracellular HDV RNA signal (Fig. 2B-D). As expected, treatments of cells with omeprazole did not show antiviral properties (Fig. 2E). To rule out the possibility of a direct effect of the drugs on viral particles, $\mathrm{HDV}_{\text {LMS }}$-containing supernatants were incubated with each drug at $50 \mu \mathrm{M}$ for $20 \mathrm{~h}$, then diluted 10 -fold in drug-free or $50 \mu \mathrm{M}$ drug-containing medium, and added to cells for $20 \mathrm{~h}$ (Fig. 3). Non-treated $\mathrm{HDV}_{\text {LMS }}$ was used as a positive control. Postinoculation, cells were washed and maintained in culture for 6 days prior to HDV RNA quantification. Results show that dilution of the drugs prior to cell inoculation lead to infection at a level comparable to that of non-treated particles, as opposed to samples with a final concentration of $50 \mu \mathrm{M}$ (Fig. 3B-D). These findings demonstrate that the drugs are efficient only when present during inoculation (Fig. 2), but they do not affect virus particles directly (Fig. 3). Additionally, the rapid wearing off of the drug effects upon washings (Fig. 2) is in favour of a direct effect on the receptor rather than an indirect effect on cell metabolism. 

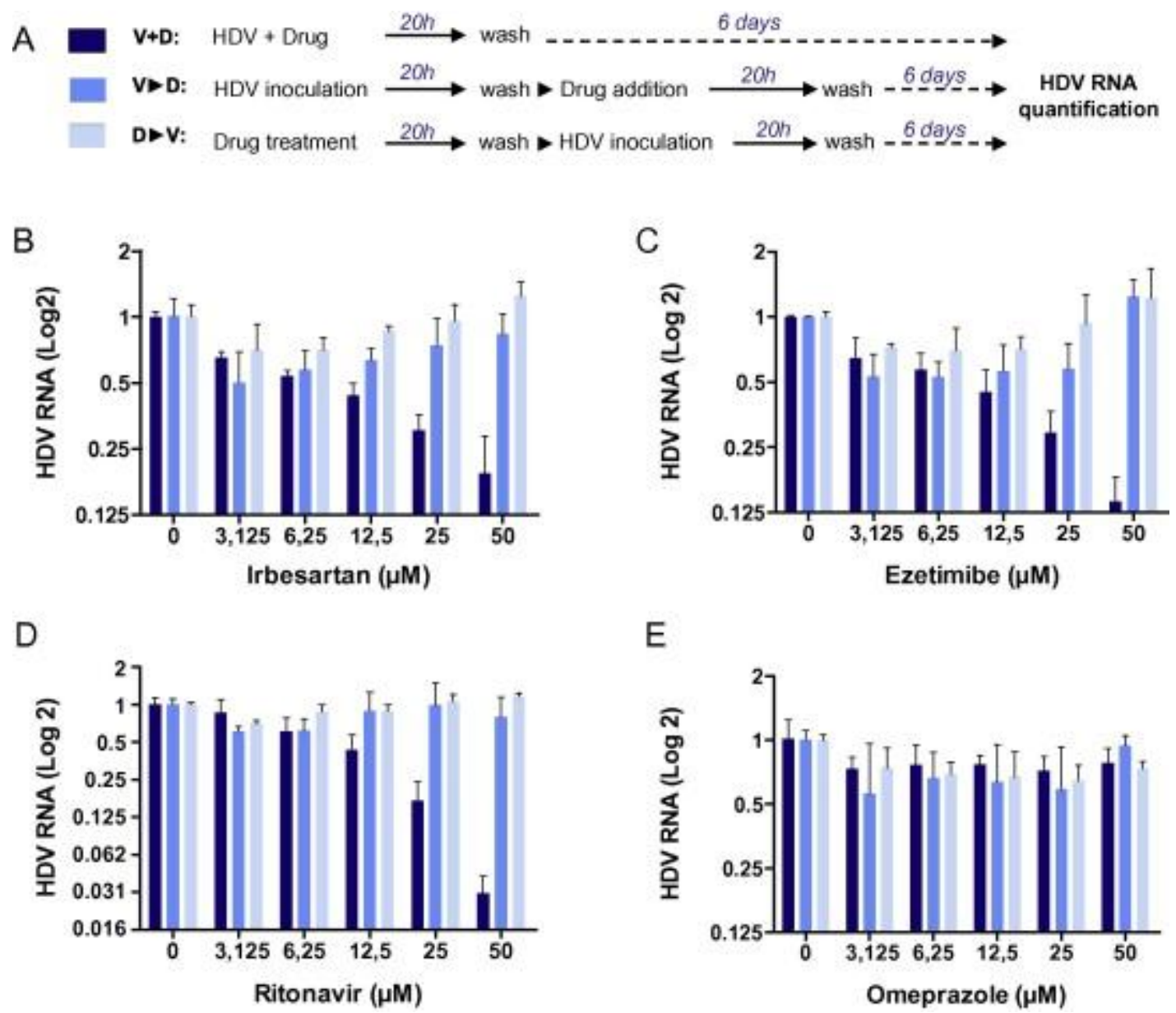

Fig. 2.

Irbesartan, ezetimibe, and ritonavir block HDV infection of Huh7/hNTCP cells. (A) Schematic representation of cell treatment is indicated. (B-E) Drugs were added to Huh7/hNTCP cells for $20 \mathrm{~h}$ at the time of viral inoculation ( $\mathrm{V}+\mathrm{D}$; dark blue), before inoculation ( $D \bullet V$; medium bleu), or after inoculation ( $\vee \triangleright D$; light blue) as described in $(A)$. After treatment, cells were washed and maintained in culture for 6 days. Intracellular HDV RNA concentration was evaluated by RT-qPCR (Taqman). Quantifications are presented relative to the non-treated samples, and compile three independent experiments. (For interpretation of the references to colour in this figure legend, the reader is referred to the web version of this article.) 
A

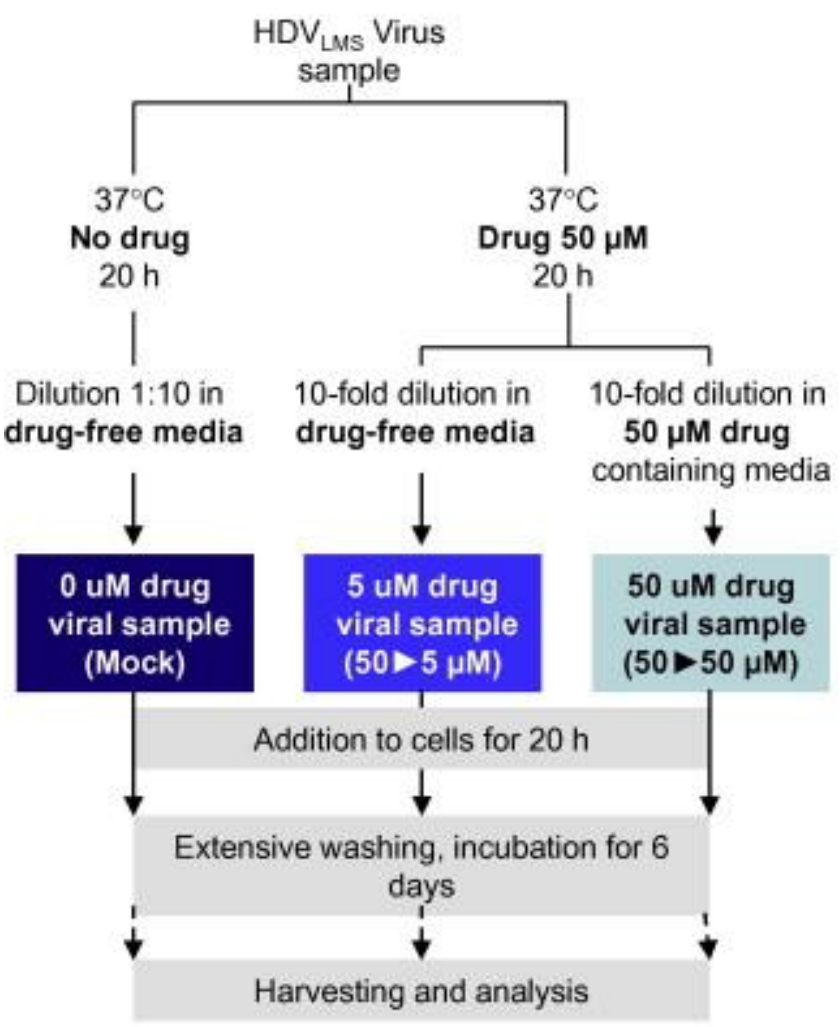

B

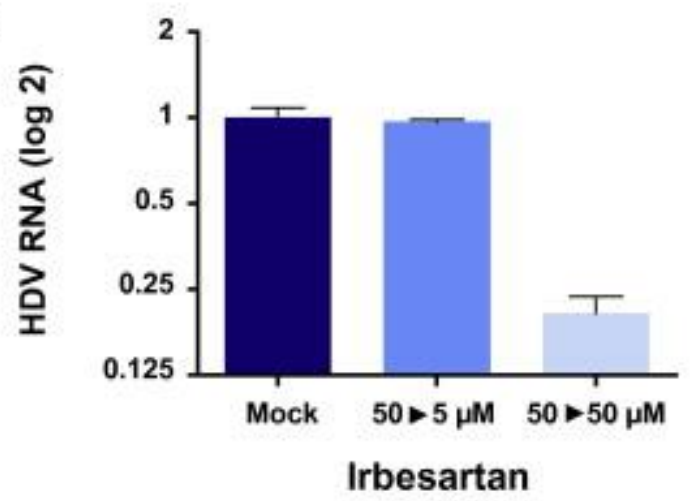

C

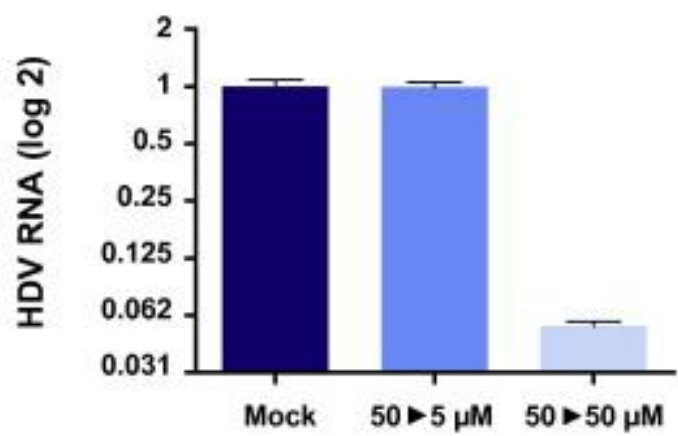

Ezetimibe

D

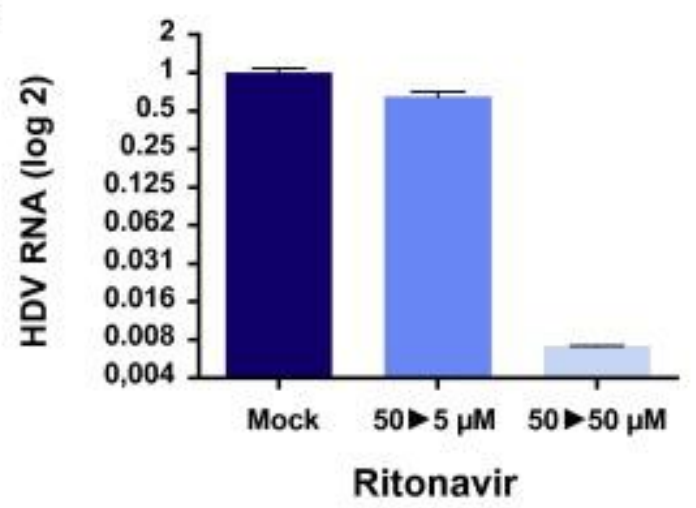

Fig. 3.

Irbesartan, ezetimibe, and ritonavir do not impair HDV infectivity but reduce susceptibility of Huh7/hNTCP to infection. (A) Schematic representation of cell treatment is indicated.

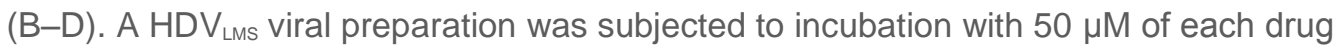
for $20 \mathrm{~h}$, and diluted 10-fold in drug-free media (50-5 $\mu \mathrm{M}$; medium blue) or in $50 \mu \mathrm{M}$ containing media (50-50 $\mu \mathrm{M}$; light blue) and added to Huh7/hNTCP for $20 \mathrm{~h}(\mathrm{~A})$. A viral sample without drug treatment was used as a positive control (Mock; dark blue). Cells were extensively washed and maintained in culture for 6 days. Intracellular HDV RNA was quantified for each condition by RT-qPCR (Taqman). Quantifications are presented relative to the non-treated samples, and compile three independent experiments. (For 
interpretation of the references to colour in this figure legend, the reader is referred to the web version of this article.)

Figure options

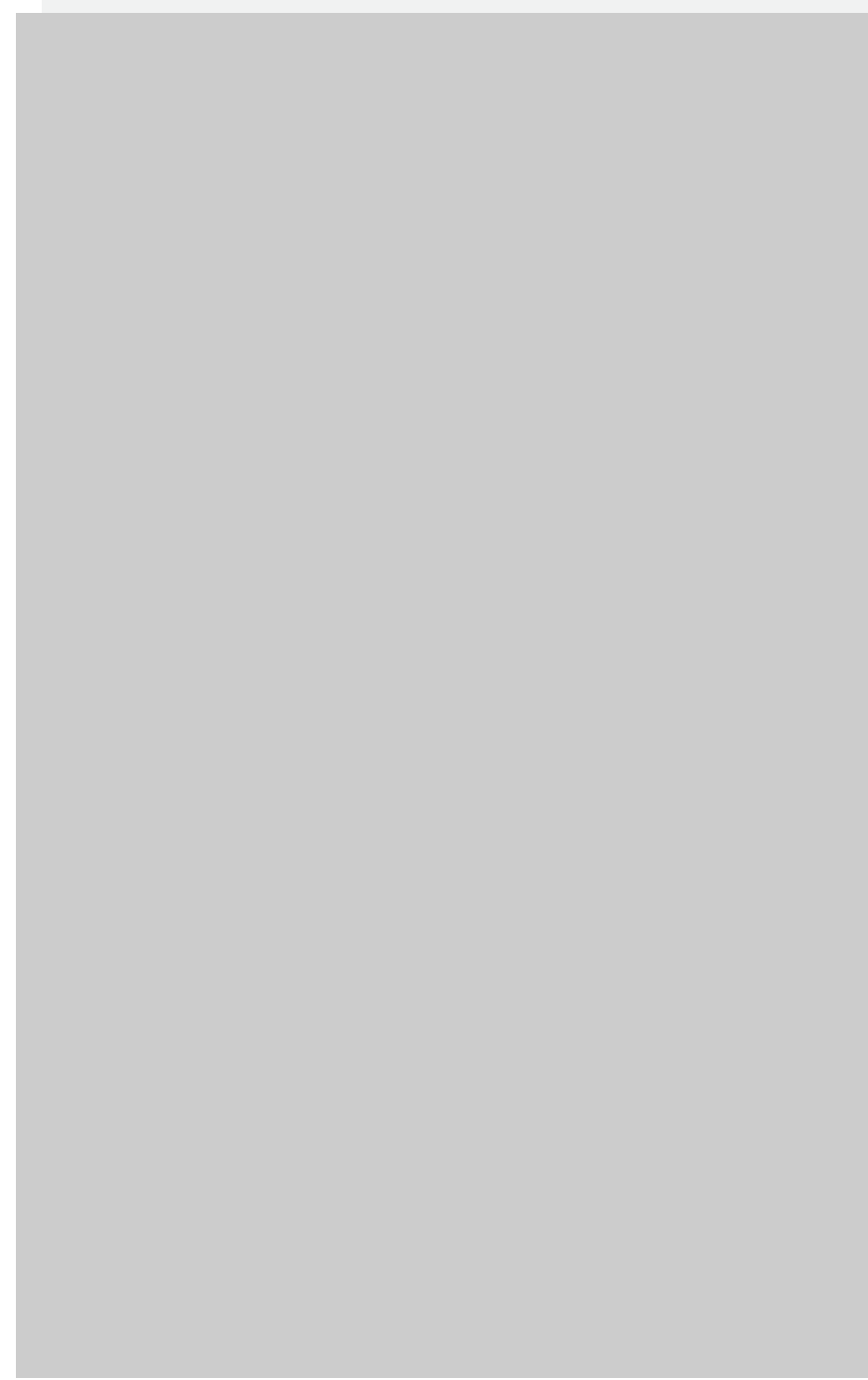

Fig. 4.

Irbesartan, ezetimibe, and ritonavir antiviral effects are additive and do not affect cellular hNTCP mRNA expression. Combinations of drugs (individual concentrations of $25 \mu \mathrm{M}$ ) were added to Huh7/hNTCP cells for $20 \mathrm{~h}$ at the time of viral inoculation, washed and maintained in culture for 6 days. Total intracellular RNA was tested for viral RNA (A) and hNTCP mRNA (B).

Figure options

Taken together, our results strongly suggest that irbesartan, ezetimibe and ritonavir prevent infection at an early stage of the viral lifecycle. A recent study suggested that ezetimibe would prevent the establishment of HBV infection in HepaRG cells at a post virus-receptor interaction step (Lucifora 
et al., 2013), but when this study was conducted, the HBV receptor had not been identified, and therefore, the ability of ezetimibe to impair hNTCP could not be addressed (Dong et al., 2013). Based on our results, we propose that the anti HBV effect of ezetimide is mainly the result of its inhibitory effect on hNTCP at viral entry. It is worth mentioning that HDV and $\mathrm{HBV}$ virions are assumed to engage the same receptors at viral entry (Blanchet and Sureau, 2007 and Engelke et al., 2006), and it is very likely that the pathways used by HDV and HBV to deliver their respective RNP or nucleocapsid cargoes to the nucleus are distinct and not equally affected by a post entry inhibitors. Thus, the logical consensus of the results obtained with ezetimibe on HBV (Lucifora et al., 2013) and HDV (present study) infections, would be that ezetimibe be active at a step between virusreceptor interaction and internalisation. Concerning ritonavir, two clinical studies (Carr and Cooper, 1997 and Velasco et al., 1999) have shown an improvement of HBV chronic carriers conditions under drug treatment. In both cases, patients were chronically coinfected with HIV, and the treatment resulted in a drop of HIV load concomitant with an increase of CD4 and CD8 cell counts and a flare of hepatitis. Several months post treatment, serum HBV DNA concentrations were dramatically decreased. HBV pathology is known to be mediated by the targeting of infected hepatocytes by the immune system. Liver damages are thus reduced in the context coinfection with HIV (Carr and Cooper, 1997). Authors hypothesised that a flare of hepatitis was the consequence of an improved host immune response triggered by a reduction in HIV viremia rather than a toxicity due to the ritonavir treatment. They additionally hypothesised that the following HBV viremia drop was the result of the improved condition of the immune system. While we agree with this hypothesis, our results strongly suggest an additional and significant contribution of ritonavir treatment at viral entry for HBV and HDV.

Altogether, we have identified three FDA approved therapeutics with inhibitory potential on the metabolic function of hNTCP that have potent antiviral effect on HDV. NTCP being the receptor for both HBV and HDV, we hypothesise that these chemicals could also exert anti-HBV effects. No significant toxicity of the compounds was observed at concentrations exhibiting antiviral properties. While we state that targeting hNTCP is most likely to account for the antiviral properties of these chemicals, we 
acknowledge that additional cellular targets might be involved. In vivo experiments are needed to determine the anti-HDV/HBV efficiency of these FDA approved compounds at doses that are already recommended in clinical practise.

\section{Acknowledgments}

We would like to thank Guillaume Castel for helpful discussions and Christine Kleinert for carefully reading the manuscript. P.L. is supported by a CIHR Grant \# MOP 93792 and received a salary support from Le Fonds de recherche du Québec (FRSQ). M.B. was supported by a postdoctoral fellowship from "La fondation Universitaire Armand-Frappier". 